\title{
Protective effect of vitamin $E$ on methyl methanesulfonate-induced teratozoospermia in adult Sprague-Dawley rats
}

\author{
ZHIAN TANG ${ }^{1 *}$, WEILIANG DING ${ }^{2 *}$, LUN WANG $^{3}$, WENCHU JIANG ${ }^{3}$, QUANXIANG ZHANG $^{2}$, \\ HONG CHEN $^{4}$, HONGNAN ZOU ${ }^{2}$, YONGKANG DONG ${ }^{2}$, JIANWEI SHAO ${ }^{2}$ and TIELIANG MA ${ }^{5}$ \\ Departments of ${ }^{1}$ Traditional Chinese Medicine, ${ }^{2}$ Laboratory, ${ }^{3}$ Pathology and ${ }^{4}$ Oncology; ${ }^{5}$ Central Laboratory, \\ The Affiliated Yixing Hospital of Jiangsu University, Yixing, Jiangsu 214200, P.R. China
}

Received August 25, 2014; Accepted May 1, 2015

DOI: $10.3892 / \mathrm{mmr} .2015 .3916$

\begin{abstract}
The protective effect of vitamin E (VE, $\alpha$-tocopherol) on methyl methanesulfonate (MMS)-induced teratozoospermia was investigated in adult rats. Rats $(\mathrm{n}=6$ per group) were divided into three groups: i) Control group, treated with distilled water from days 1 to 5 ; ii) the MMS group, treated with MMS at a dose of $40 \mathrm{mg} \cdot \mathrm{kg}^{-1}$ from days 1-5; or iii) the VE+MMS group, treated with MMS at a dose of $40 \mathrm{mg} \cdot \mathrm{kg}^{-1}$ from days $1-5$, followed by VE at a dose of $150 \mathrm{mg} \cdot \mathrm{kg}^{-1}$ from day 6 for 6 weeks. Sperm count, motility and morphology were examined following treatment with VE. The serum testosterone level and antioxidant enzyme activity were measured, and the localization of Vasa, promyelocytic leukemia zinc finger protein (Plzf) and synaptonemal complex protein 3 (Scp3) were also examined. MMS treatment decreased sperm count and motility, and the levels of immunoreactive serum testosterone and endogenous antioxidants. In addition, MMS increased the percentage of abnormal sperm and the levels of free radicals. After MMS and VE treatment, sperm count and motility were significantly higher in rats from the VE+MMS group than in the MMS group. In addition, the serum testosterone concentration, as well as the levels of Vasa and free radicals and the percentage of abnormal sperm, decreased. The results indicated that VE has protective effects against MMS-induced teratozoospermia in adult rats.
\end{abstract}

Correspondence to: Dr Tieliang Ma, Central Laboratory, The Affiliated Yixing Hospital of Jiangsu University, 75 Tongzhenguan Road, Yixing, Jiangsu 214200, P.R. China

E-mail: matieliang@foxmail.com

${ }^{*}$ Contributed equally

Key words: methyl methanesulfonate, vitamin E, teratozoospermia, rat

\section{Introduction}

Methyl methanesulfonate (MMS) exerts serious genotoxic effects, including induction of DNA damage, and micronuclei and cell cycle alterations. MMS, a potent alkylating agent, is a testicular toxicant that damages germ cell DNA, thereby affecting sperm morphology in mice (1) and rats (2,3). MMS also disrupts the differentiation of germ cells into sperm cells $(4,5)$. Oxidative stress is an imbalance between the systemic manifestation of reactive oxygen species (ROS) and the biological system's ability to detoxify reactive intermediates or to repair the resulting damage efficiently (6). The testis contains a high level of polyunsaturated membrane lipids; thus, it is a target of oxidative stress (7). Nevertheless, organisms have developed numerous defense mechanisms to protect themselves from damage caused by ROS. For example, antioxidants, such as VE can scavenge free radicals $(8,9)$ and arrest the lipid peroxidation of lipoproteins that are present within biological membranes (10). VE is relatively abundant in the testis, where it protects sperm from ROS and inhibits lipid peroxidation (11). Any deficiency in VE may result in testicular damage, degenerated seminiferous tubules, and defective germ cells (12). By contrast, an increase in the level of VE has been shown to have an opposite effect, that is, to increase total sperm output in rabbits (13) and sheep (14). A previous study has shown VE to have a protective effect on pesticide-induced oxidative stress (13). Thus, the goal of this study was to investigate the protective role of $\mathrm{VE}$ on MMS-induced teratozoospermia in adult rats. Therefore, the effects of VE in adult rats treated with MMS was compared with the effects of treatment with MMS alone.

\section{Materials and methods}

Experimental animals. Eighteen adult male Sprague-Dawley (SD) outbred rats at 8 weeks of age and weighing 210-230 g were obtained from a closed random bred colony at the Animal Center of Nanjing Medical University. Animals were housed under standard conditions of $21 \pm 2^{\circ} \mathrm{C}$ and a $12 \mathrm{~h}$ light/dark cycle with access to food and water ad libitum. Animals received humane care in compliance with the guidelines of 
the National Institutes of Health. This study was approved by the Institutional Animal Care and Use Committee of Jiangsu University (Yixing, China).

Treatment of animals with MMS. Rats were randomly divided into three groups ( $n=6$ per group) as follows: i) The control group, treated with distilled water from days 1 to 5; ii) The MMS group, treated with MMS at a dose of $40 \mathrm{mg} \cdot \mathrm{kg}^{-1}$ (Sigma-Aldrich, St. Louis, MO, USA) from day 1-5; and iii) the VE+MMS group, treated with MMS at a dose of $40 \mathrm{mg} \cdot \mathrm{kg}^{-1}$ from days $1-5$, followed by VE (Sigma-Aldrich, St. Louis, MO, USA) at a dose of $150 \mathrm{mg} \cdot \mathrm{kg}^{-1}$ from day 6 for 6 weeks. All experimental agents were delivered by gavage using a non-flexible stainless steel feeding tube (Sangon Biotech Co., Ltd., Shanghai, China). Thereafter, rats were anesthetized with $5 \%$ chloral hydrate $(1.5 \mathrm{ml})$, and blood was collected by cardiac puncture into standard test tubes after the rats had been anaesthetized. Samples were centrifuged at $500 \mathrm{x} \mathrm{g}$ for $15 \mathrm{~min}$ at $4^{\circ} \mathrm{C}$ to obtain serum and then stored at $-40^{\circ} \mathrm{C}$. Testes and epididymis were quickly excised and suspended in ice-cold phosphate buffered saline (PBS). One testis from one rat was fixed in $10 \%$ formaldehyde (w/v) and the other testis was stored at $-80^{\circ} \mathrm{C}$. Sperm count, motility and morphology of the three groups were examined following treatment with VE.

Counting of epididymal sperm. The epididymis was weighed prior to sperm counting. Epididymal spermatozoids were counted as previously described with minor modifications to the protocol (15). In brief, one of the epididymis was minced in $5 \mathrm{ml} \mathrm{PBS}$ ( $\mathrm{pH} 7.4$ ), placed on an orbital shaker (Sangon Biotech Co., Ltd.) for $10 \mathrm{~min}$, and incubated at room temperature for $2 \mathrm{~min}$. Semen (25 $\mu \mathrm{l})$ was transferred to $1 \mathrm{ml}$ fixative, and spermatozoids were counted under a light microscope (Olympus BX41; Olympus Corporation, Tokyo, Japan) and using a hemocytometer (Sangon Biotech Co., Ltd.). The other epididymis was stored at $-80^{\circ} \mathrm{C}$.

Sperm motility. Approximately 200 spermatozoids with progressive motility from each epididymis were evaluated under a light microscope within 2-4 min after they were isolated as previously described (16).

Detection of sperm abnormalities. Fixed spermatozoids were smeared onto a slide glass, air-dried overnight and stained with the Diff-Quick kit (Baso Diagnostics, Inc., Zhuhai, China) (17). Approximately 300 spermatozoids from each treatment group were counted under a light microscope, and the percentages of morphologically abnormal spermatozoids (detached head and/or coiled tail) were recorded as previously described (1).

Measurement of the serum testosterone level. The serum testosterone level was measured as previously described (18) using a commercially available chemiluminescence-linked immunoassay (Beckman-Coulter UniCel DxI 800, Beckman-Coulter, Brea, CA, USA).

Antioxidant enzyme activity and oxidative stress assays. Different oxidative stress indicators, including free radicals
Table I. Immunohistochemical staining by comparison of the average IOD values.

\begin{tabular}{lccc}
\hline Group & Vasa & Plzf & Scp3 \\
\hline Control & $0.18 \pm 0.04$ & $0.18 \pm 0.05$ & $0.18 \pm 0.06$ \\
MMS & $0.12 \pm 0.03^{\mathrm{a}}$ & $0.20 \pm 0.04$ & $0.17 \pm 0.07$ \\
MMS+VE & $0.21 \pm 0.04^{\mathrm{b}}$ & $0.22 \pm 0.02$ & $0.19 \pm 0.05$ \\
\hline
\end{tabular}

Data are presented as the mean \pm standard error of the mean, $n=15$. ${ }^{\mathrm{a}} \mathrm{P}<0.05$, compared with the control group an ${ }^{\mathrm{b}} \mathrm{P}<0.05$, compared with the MMS group. One way analysis of variance test. IOD, integrated optical density; Plzf, promyelocytic leukemia zinc finger protein; Scp3, synaptonemal complex protein 3; MMS, methyl methanesulfonate; VE, vitamin $\mathrm{E}$.

(e.g., superoxide anion and hydroxyl radical) and antioxidants [e.g., superoxide dismutase (SOD) and glutathione peroxidase (GSH-Px)], were measured in sera from rats in each treatment group. All assays were performed according to the instructions provided in the kits (Jiancheng Bioengineering Research Institute, Nanjing, China).

Immunohistochemistry. Testes were fixed in $10 \%$ formaldehyde $(\mathrm{w} / \mathrm{v})$ for at least $24 \mathrm{~h}$ and then processed for paraffin embedding (19). Rabbit polyclonal anti-Vasa (cat. no. ab13840), rabbit polyclonal anti-promyelocytic leukemia zinc finger protein (Plzf; cat. no. ab39354), and rabbit polyclonal anti-synaptonemal complex protein 3 (Sycp3; cat. no. ab150292) antibodies were purchased from Abcam (Cambridge, UK). Anti-mouse IgG (H+L, alkaline conjugate) was purchased from Promega Corporation (Madison, WI, USA). Sections (5 $\mu \mathrm{m})$ were deparaffinized in xylene, rehydrated in a gradient series of alcohol, and boiled in antigen retrieval solution (Sangon Biotech Co., Ltd.) at $95^{\circ} \mathrm{C}$ for $10 \mathrm{~min}$. The sections were treated with $3 \%$ hydrogen peroxide (v/v) for $10 \mathrm{~min}$, blocked with $3 \%$ bovine serum albumin (v/v) (Sangon Biotech Co., Ltd.) and 10\% normal donkey serum (v/v) (Sangon Biotech Co., Ltd.) in Tris-buffered saline (TBS), and then incubated with antibodies overnight at $4^{\circ} \mathrm{C}$. Thereafter, sections were washed with TBS and incubated with a biotinylated and streptomycin-labeled goat anti-mouse antibody (cat. no. KIT-5010; Maixin Bio, Ltd., Fujian, China) for $15 \mathrm{~min}$ at room temperature. Immunoreactive proteins were visualized with 3,3'-diaminobenzidine tetrahydrochloride. Sections were examined under a light microscope (Eclipse 80i; Nikon, Tokyo, Japan). The expression levels of protein were measured and demonstrated by the Integrated Optical Density (IOD) values using Image-Pro Plus 6.0 software (Media Cybernetics, Inc., Rockville, MD, USA).

Statistical analysis. SPSS 14.0 (SPSS Inc., Chicago, IL, USA) statistical software for Windows was used. Results are presented as the mean \pm standard deviation. One-way analysis of variance (followed by Tukey's post hoc test) or Mann-Whitney U-test was used to compare the means across different treatment groups. $\mathrm{P}<0.05$ was considered to indicate a statistically significant difference. 

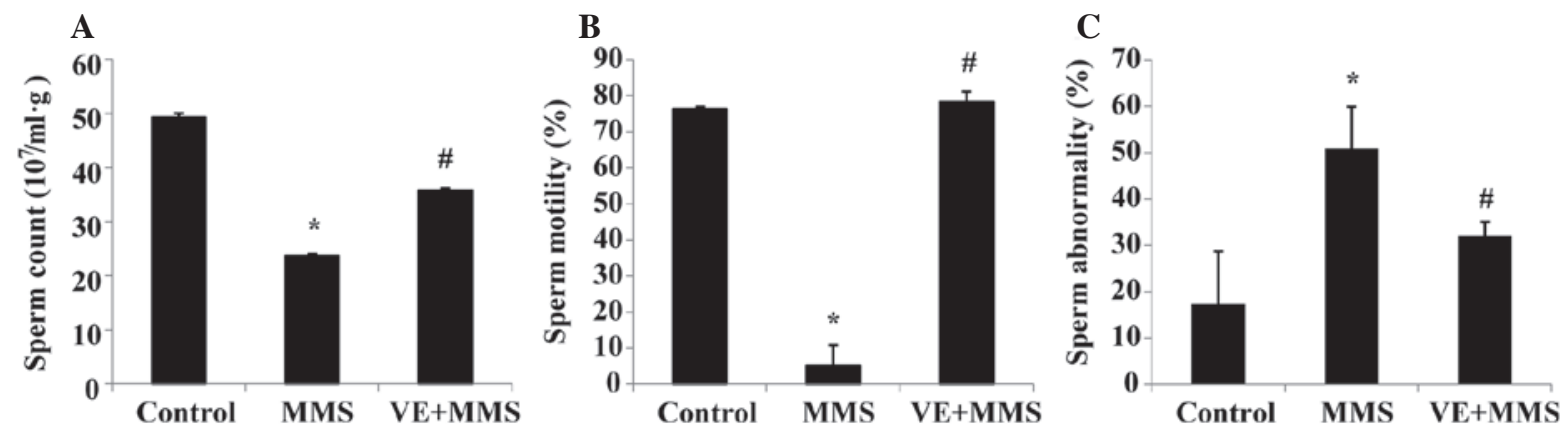

Figure 1. Effects of MMS and VE on sperm characteristics in male rats. (A) Sperm count. (B) Sperm motility. (C) Sperm abnormalities. "P<0.05, compared with the control group and ${ }^{*} \mathrm{P}<0.05$, compared with the MMS group. A one way analysis of variance test was used for the statistical analysis of $\mathrm{A}$, and a Mann-Whitney U test for B and C. MMS, methyl methanesulfonate; VE, vitamin E.

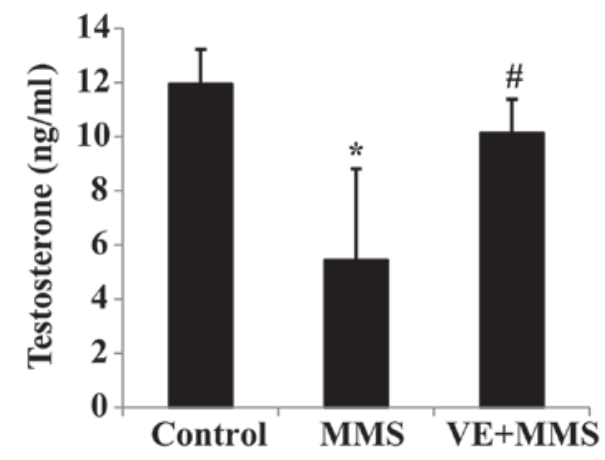

Figure 2. Effects of MMS and VE on the serum testosterone level in male rats. ${ }^{*} \mathrm{P}<0.05$, compared with the control group; ${ }^{*} \mathrm{P}<0.05$, compared with the MMS group, as determined by a one way analysis of variance test. MMS, methyl methanesulfonate; VE, vitamin E.

\section{Results}

Sperm characteristics. Sperm count $\left(2.36 \pm 0.04 \times 10^{8}\right)$ and motility $(5.0 \pm 5.8 \%)$ decreased significantly $(\mathrm{P}<0.05)$ in rats from the MMS group compared with those from the control group $\left(4.92 \pm 0.07 \times 10^{8}\right.$ and $76.3 \pm 0.7 \%$, respectively). However, there was an increase in the percentage of abnormal sperm $(50.9 \pm 9.0 \%)$ compared with the control group $(17.3 \pm 11.4 \%)$. After MMS and VE treatment, sperm count $\left(3.57 \pm 0.04 \times 10^{8}\right)$ and motility $(78.3 \pm 2.9 \%)$ were significantly higher $(\mathrm{P}<0.05)$ in rats from the VE+MMS group than in the MMS group (Fig. 1A and B). However, there was a significant decrease $(\mathrm{P}<0.05)$ in the percentage of abnormal sperm in rats from the VE+MMS group $(32.0 \pm 3.0 \%)$ compared with the MMS group (Fig. 1C).

Levels of serum testosterone. There was a significant decrease $(\mathrm{P}<0.05)$ in the serum testosterone level in the MMS group $(5.4 \pm 3.4 \mathrm{ng} / \mathrm{ml})$ compared with the control $(11.9 \pm 1.3 \mathrm{ng} / \mathrm{ml})$ and a significant increase in the VE+MMS group $(10.1 \pm 1.3 \mathrm{ng} / \mathrm{ml})$ compared with the MMS group (Fig. 2).

Serum oxidative stress indicators. The levels of hydroxyl and superoxide free radicals were significantly higher in the MMS group, as compared with the control group; and lower in the VE+MMS group, as compared with the control group
(Fig. 3A and B). By contrast, the levels of antioxidants SOD and GSH-Px, were lower in the MMS group than in the control and VE+MMS groups (Fig. 3C and D).

Immunohistochemical findings. The histo-architecture of the testes from control rats was normal, consisting of uniform, well-organized seminiferous tubules with complete spermatogenesis and normal interstitial connective tissue (Fig. 4). By contrast, testes from rats treated with MMS showed seminiferous tubule degeneration manifested by shrunken, disorganized tubules with irregular, buckled basement membranes and incomplete spermatogenesis (Fig. 4). Moreover, the seminiferous tubules were virtually devoid of spermatids and sperm. After MMS+VE treatment, there was an improvement in spermatogenesis, demonstrated by the presence of elongated spermatids and sperm in the majority of the seminiferous tubules (Fig. 4).

As observed using immunohistochemistry, the level of Vasa, a biomarker of germ cells, decreased in the testis of rats treated with MMS; however, its level increased in rats treated with VE+MMS compared with those treated with MMS alone (Fig. 5). There were no changes in Plzf and Sycp3, biomarkers of spermatogonial stem cells and spermatocytes, respectively, following MMS and MSS+VE treatment (Table I).

\section{Discussion}

Previous studies have shown MMS to decrease sperm number and affect sperm head morphology in mice and rats (1-3). Although VE can protect cells from damage caused by free radicals and oxidative products, there are few studies on the protective effect of VE in animals previously exposed to MMS $(8,10,12-14)$. The present study demonstrated that VE partially alleviated the damage caused by MMS in the testis.

Ozawa et al (2) demonstrated that body, testis and epididymis weights, as well as food consumption decrease following administration of MMS. Germ cell exfoliation, Sertoli cell vacuolization, and epididymal duct cell debris were also observed (2). Although VE does not protect the testes from acrylamide toxicity, treatment with VE following cessation of acrylamide treatment improves recovery (20). In the present study, MMS treatment resulted in seminiferous tubule degeneration, and VE treatment improved the recovery of spermatogenesis. 
A

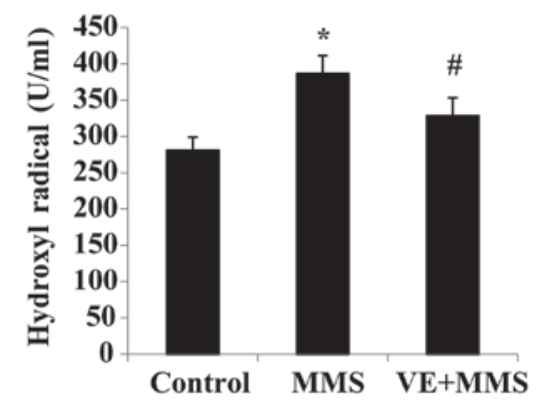

C

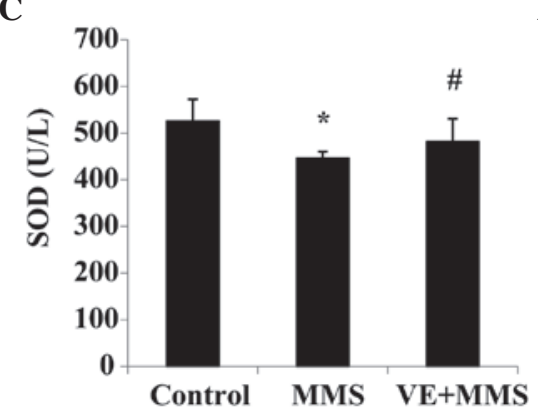

B

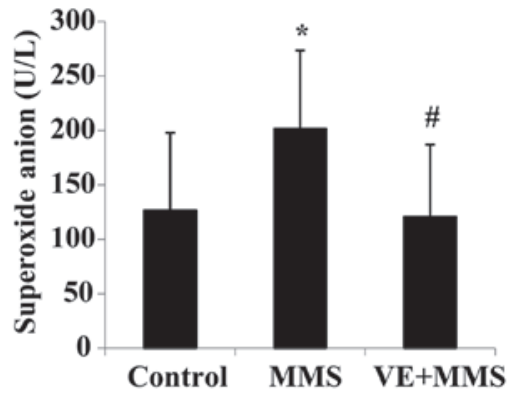

D

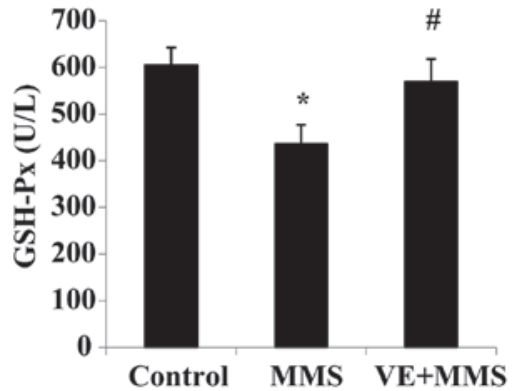

Figure 3. Effects of MMS and VE on antioxidant enzyme activities in male rats. (A) Level of the hydroxyl radical. (B) Level of the superoxide anion. (C) Level of SOD. (D) Level of GSH-Px. " P $<0.05$, compared with the control group; ${ }^{*} \mathrm{P}<0.05$ compared with the MMS group. One way analysis of variance test. MMS, methyl methanesulfonate; VE, vitamin E; SOD, superoxide dismutase; GSH-Px, glutathione peroxidase.

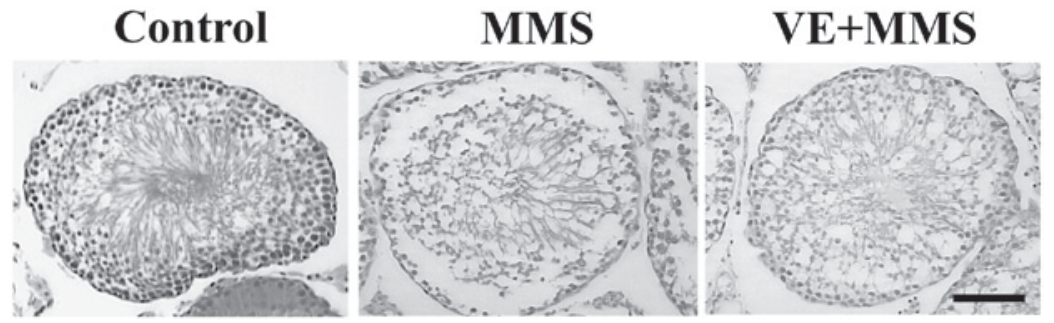

Figure 4. Structure of the testis of different groups. Scale bar, $50 \mu \mathrm{m}$. MMS, methyl methanesulfonate; VE, vitamin E.

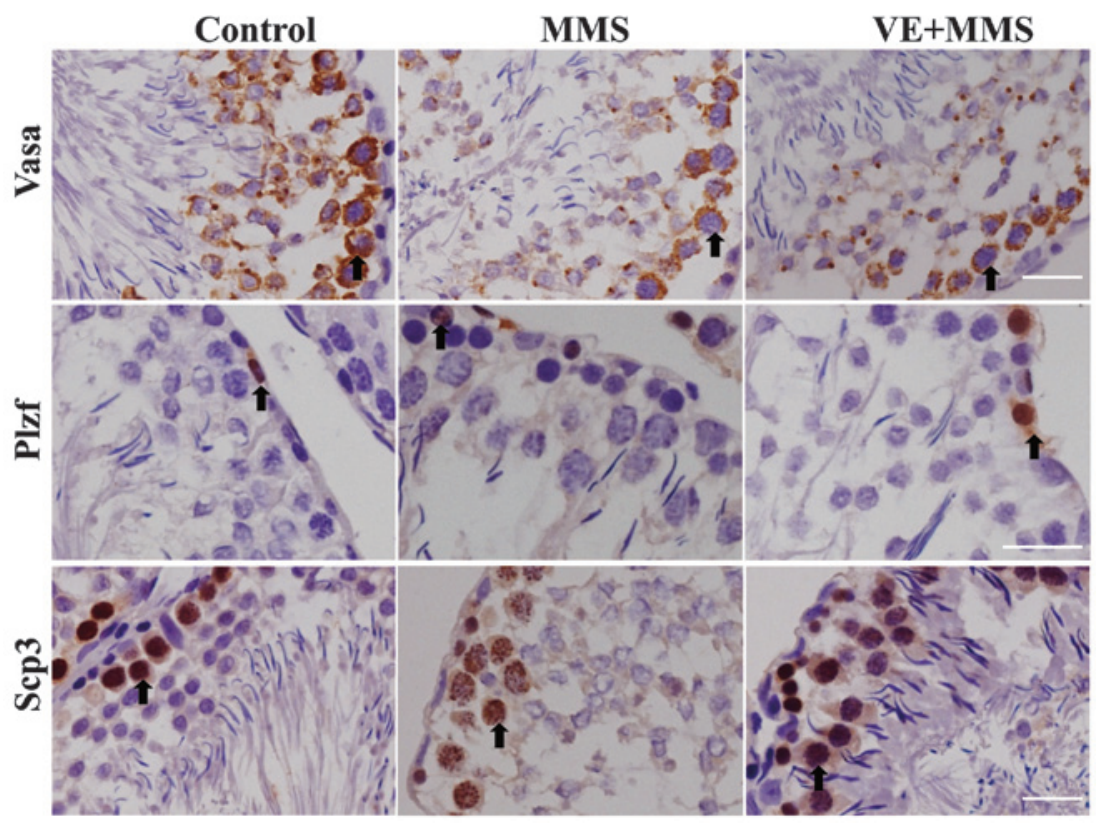

Figure 5. Immunolocalization of Vasa, Plzf, and Scp3 in the three treatment groups. Arrows point to protein localization. Scale bar, $50 \mu \mathrm{m}$. MMS, methyl methanesulfonate; VE, vitamin E; Plzf, promyelocytic leukemia zinc finger protein; Scp3, synaptonemal complex protein 3. 
VE can protect critical cellular structures from free radical and oxidative damage (21). As a soluble, lipid-based antioxidant, $\mathrm{VE}$ is protective against oxidative stress by preventing the production of lipid peroxides and scavenging free radicals (11). $\mathrm{VE}$ is also important in maintaining the function of the testis, epididymis and accessory glands (22), possibly by improving sperm quality and quantity. For example, VE improved semen quality and quantity in humans (11), sheep (12), and chickens (22). VE has also been demonstrated to protect rat testis against experimental cryptorchidism $(23,24)$. Furthermore, the increased free radicals generated by acrylamide exposure in the testes may have been scavenged in the testes during recovery period (20). MMS at doses of $20-40 \mathrm{mg} / \mathrm{kg}$ for two consecutive weeks increased heme oxygenase-1 (HO-1) mRNA and protein in the rat testis (25). In this study, MMS increased free radical formation, which decreased following VE treatment, while the levels of two endogenous antioxidants indicators (SOD and GSH-Px) showed an opposite tendency. These results indicate that MMS induces oxidative stress in adult rats, and VE has the capability of scavenge them.

MMS damages germ cell DNA by creating lesions within the DNA, which can disrupt the differentiation of germ cells into spermatozoa (5). This is in agreement with the results of the present study, which showed an increase in the percentage of defective spermatozoids. Moreover, the level of Vasa decreased after MMS treatment, while the levels of Plzf and Sycp3 remained unchanged, suggesting that MMS has little influence on spermatogonial stem cells and spermatocytes. Additional experiments are requried to address the mechanism of MMS action in the adult testis.

In conclusion, MMS treatment of adult rats affects germ cells in the testis, and VE may aid in repairing the damage caused by oxidative stress.

\section{Acknowledgements}

This study was supported by the Fund of Science and Technology of Yixing (grant no. 2013-15) and Development Fund of Clinical Science and Technology (grant no. JLY20120070, 2012, Jiangsu University).

\section{References}

1. Wyrobek AJ and Bruce WR: Chemical induction of sperm abnormalities in mice. Proc Natl Acad Sci USA 72: 4425-4429, 1975.

2. Ozawa S, Yokoi R, Kitamura T, Kuriyama K, Kobayashi K and Shibata N: Collaborative work to evaluate toxicity on male reproductive organs by repeated dose studies in rats 15). Two-week and 4-week administration study of methyl methanesulfonate (MMS). J Toxicol Sci 25: 155-162, 2000.

3. Cassidy SL, Dix KM and Jenkins T: Evaluation of a testicular sperm head counting technique using rats exposed to dimethoxyethyl phthalate (DMEP), glycerol alpha-monochlorohydrin $(\mathrm{GMCH})$, epichlorohydrin (ECH), formaldehyde (FA), or methyl methanesulphonate (MMS). Arch Toxicol 53: 71-78, 1983.

4. Bruce WR and Heddle JA: The mutagenic activity of 61 agents as determined by the micronucleus, Salmonella, and sperm abnormality assays. Can J Genet Cytol 21: 319-334, 1979.
5. Inoue M, Kurihara T, Yamashita M and Tatsumi K: Effects of treatment with methyl methanesulfonate during meiotic and postmeiotic stages and maturation of spermatozoa in mice. Mutat Res 294: 179-186, 1993

6. Ochsendorf FR: Infections in the male genital tract and reactive oxygen species. Hum Reprod Update 5: 399-420, 1999.

7. Aitken RJ, Harkiss D and Buckingham DW: Analysis of lipid peroxidation mechanisms in human spermatozoa. Mol Reprod Dev 35: 302-315, 1993.

8. Ingold KU, Burton GW, Foster DO, et al: A new vitamin E analogue more active than alpha-tocopherol in the rat curative myopathy bioassay. FEBS Lett 205: 117-120, 1986.

9. Jones DP, Kagan VE, Aust SD, Reed DJ and Omaye ST: Impact of nutrients on cellular lipid peroxidation and antioxidant defense system. Fundam Appl Toxicol 26: 1-7, 1995.

10. Sheridan PA and Beck MA: The immune response to herpes simplex virus encephalitis in mice is modulated by dietary vitamin E. J Nutr 138: 130-137, 2008.

11. Akiyama M: In vivo scavenging effect of ethylcysteine on reactive oxygen species in human semen. Nihon Hinyokika Gakkai Zasshi 90: 421-428, 1999 (In Japanese).

12. Wilson MJ, Kaye D, Smith WE, Quach HT, Sinha AA and Vatassery GT: Effect of vitamin E deficiency on the growth and secretory function of the rat prostatic complex. Exp Mol Pathol 74: 267-275, 2003.

13. Yousef MI: Vitamin E modulates reproductive toxicity of pyrethroid lambda-cyhalothrin in male rabbits. Food Chem Toxicol 48: 1152-1159, 2010.

14. Yue D, Yan L, Luo H, Xu X and Jin X: Effect of Vitamin E supplementation on semen quality and the testicular cell membranal and mitochondrial antioxidant abilities in Aohan fine-wool sheep. Anim Reprod Sci 118: 217-222, 2010.

15. Yokoi K, Uthus EO and Nielsen FH: Nickel deficiency diminishes sperm quantity and movement in rats. Biol Trace Elem Res 93: 141-154, 2003.

16. Sönmez M, Türk G and Yüce A: The effect of ascorbic acid supplementation on sperm quality, lipid peroxidation and testosterone levels of male Wistar rats. Theriogenology 63: 2063-2072, 2005.

17. Enginsu ME, Dumoulin JC, Pieters MH, Bras M, Evers JL and Geraedts JP: Evaluation of human sperm morphology using strict criteria after Diff-Quik staining: correlation of morphology with fertilization in vitro. Hum Reprod 6: 854-858, 1991.

18. PrattJJ, Woldring MGand Villerius L:Chemiluminescence-linked immunoassay. J Immunol Methods 21: 179-184, 1978.

19. Davidoff MS, Middendorff R, Pusch W, Müller D, Wichers S and Holstein AF: Sertoli and Leydig cells of the human testis express neurofilament triplet proteins. Histochem Cell Biol 111: 173-187, 1999.

20. Rahangadale S, Jangir BL, Patil M, et al: Evaluation of protective effect of vitamin e on acrylamide induced testicular toxicity in wister rats. Toxicol Int 19: 158-161, 2012.

21. Erin AN, Spirin MM, Tabidze LV and Kagan VE: Formation of alpha-tocopherol complexes with fatty acids. A hypothetical mechanism of stabilization of biomembranes by vitamin $\mathrm{E}$. Biochim Biophys Acta 774: 96-102, 1984.

22. Cerolini S, Zaniboni L, Maldjian A and Gliozzi T: Effect of docosahexaenoic acid and alpha-tocopherol enrichment in chicken sperm on semen quality, sperm lipid composition and susceptibility to peroxidation. Theriogenology 66: 877-886, 2006.

23. Vigueras-Villaseñor RM, Ojeda I, Gutierrez-Pérez O, et al: Protective effect of $\alpha$-tocopherol on damage to rat testes by experimental cryptorchidism. Int J Exp Pathol 92: 131-139, 2011.

24. Rahangadale S, Kurkure N, Prajapati B, Hedaoo V and Bhandarkar AG: Neuroprotective effect of vitamin e supplementation in wistar rat treated with acrylamide. Toxicol Int 19: 1-8, 2012.

25. Ashino T, Ozawa S, NumazawaS and Yoshida T: Tissue-dependent induction of heme oxygenase- 1 and metallothionein-1/2 by methyl methanesulfonate. J Toxicol Sci 28: 181-189, 2003. 